\title{
What Do Audiences Want from a Public Art Gallery in the Digital Age?
}

\author{
Clare Harding \\ Wrexham Glyndwr University \\ Faculty of Art Science and Technology \\ North Wales \\ Clare@clare-harding.com
}

Adrian Gradinar

Lancaster University, UK

adrian.gradinar@gmail.com

\author{
Susan Liggett \\ Wrexham Glyndwr University \\ Faculty of Art Science and Technology \\ North Wales \\ S.Liggett@glyndwr.ac.uk \\ Mark Lochrie \\ University of Central Lancashire, UK \\ MLochrie@uclan.ac.uk
}

\begin{abstract}
This paper outlines the human-centred design approach taken to create a new analytical framework to understand audiences and establish themes, patterns and behaviours at MOSTYN, a public contemporary art gallery in Llandudno, North Wales. Wrexham Glyndwr University PhD student Clare Harding collaborated with Dr Adrian Gradinar, and Dr Mark Lochrie from Media Innovation Studio, University of Central Lancashire, to test the conceptual framework with the EDGE (Experiential Display to Generate Engagement) research project that secured Innovate UK and the Arts Council of Wales funding. EDGE applied a Human Centred Design process to MOSTYN, Wales' foremost contemporary Art Gallery MOSTYN to investigate audience expectations of a public art gallery in the digital age. EDGE was designed to help MOSTYN define their purpose as a public art gallery in the face of rapidly developing, culturally competing technologies. Phase one of the project used design thinking and iterative processes to explore new and authentic ways in which MOSTYN can co-design their visitor experience with audiences. Phase two, from April 2019, will use findings to build a digital interface within the gallery to create an interactive exhibition of digital art. This will be accompanied by a six-month engagement programme to build links with new audiences and up-skill both the general public and regional artists. The scope and limitation of the research as identified so far are discussed with a focus on how human-centred design approaches were used to create a new analytical framework. The testing of lo-fi prototypes will be discussed within the gallery setting and the insights uncovered by deployment of the framework, tools and MOSTYN's engagement programme with a critical review of the methodological approach used and findings to date.
\end{abstract}

Public art gallery. Digital curation. Gallery audiences. Contemporary art. Digital interventions. Human-centred design.

\section{INTRODUCTION: THE MOSYTN CONTEXT}

MOSTYN is a public art gallery located in Llandudno town centre. MOSTYN incorporates six gallery spaces, a studio space, a meeting room that is available for private hire, a retail area and a café. It has two full time staff and the equivalent of twelve part time staff, plus a small number of volunteers. It is one of the 67 Arts Council Wales (ACW) Arts Portfolio Wales organisations. This means that all of MOSTYN's operational costs are met by ACW via an annual grant. All exhibitions and further activity are funded via other grants and charitable funds, commercial activity and visitor donations.
Entry to the gallery is free and MOSTYN has approximately 80,000 visitors a year. The gallery hosts 3 major 'flagship' seasons of international contemporary art every year. Three of the gallery spaces show a staggered exhibition of more affordable arts and crafts and the shop sells a range of merchandise. MOSTYN also runs a series of engagement events throughout the year and works with schools and families, as well as delivering public talks, children's programmes and specialist art courses. MOSTYN activities are promoted through their website (https://www.mostyn.org/) and via social media. In addition, many aspects of the gallery programme 
are promoted via local and national press, periodicals and specialist art press.

Harding joined the MOSTYN team in September 2017 as Curator of Digital Content and Media and Resident Researcher as part of a strategic partnership between MOSTYN and Wrexham Glyndwr University (WGU). Through interviewing staff and stakeholders, experimenting with digital media and monitoring social media she mapped out the existing organisational relationships within MOSTYN to analyse where digital tools could be of the greatest benefit. Once these areas were identified she sought funding for projects from Innovate UK and ACW.

\section{HUMAN CENTRED DESIGN PROCESSES}

Hartley's research work at the Whitworth Art Gallery Manchester provided a starting point for this research - in particular how he used Cultural Historical Action Theory ('CHAT', Vgotsky 1978) to look at 'the institutionalisation of museums' digital practices and online resources and sociology's particular understandings of online structures and organization" (Hartley 2014).

CHAT theory espouses that consciousness is essentially subjective and shaped by the history of each individual's social and cultural experience and that "the events that occur during the activity and the consequences of the activity can qualitatively change the participant, his/her object and motives for participation, the social environment of the activity, and the activity itself" (Kaptelinin 2005; Rogoff 1995, cited in Yamagata-Lynch 2007).

Applying CHAT theory facilitated Harding to create an organisational activity map to understand the context and origination of MOSTYN's identified issues and their dialectical relationships. Such an approach is condoned by Yamagata-Lynch who said that "although [activity systems analysis] cannot solve all challenges in design-based research, it can provide an initial framework for making sense of complex data sets to find systematic implications that inform both theory and practice" (Yamagata-Lynch 2007).

As Kaptelinin identifies "the key advantage of activity theory appears to be in supporting researchers and practitioners in their own inquiryfor instance, by helping to ask right questionsrather than providing ready-made answers" (Kaptelinin 2005 cited in Yamagata-Lynch 2007).

Later he says that "Adopting an activity-theoretical perspective has an immediate implication for design: it suggests that the primary concern of designers of interactive systems should be supporting meaningful human activities in everyday contexts, rather than striving for logical consistency and technological sophistication" (Kaptelinin 2005 cited in Yamagata-Lynch 2007).

For MOSTYN a hybrid approach was taken utilising Cultural Historical Action Theory.

\subsection{Cultural historical action theory}

According to this theoretical approach, MOSTYN is the Subject, both in terms of its physicality (building) and its defining features (contemporary art gallery).

The Object is the motive of MOSTYN's activity and how these are aligned with strategic objectives from the MOSTYN business plan. However, it's also the way that these Objects are achieved (e.g. brand standards, insistence on bilingual information, the curatorial position) - the choices MOSTYN staff and stakeholders make to fulfil the objectives.

This is why this research needed to be primarily a human activity study - there is a need to understand behaviour within the MOSTYN setting to understand why outcomes are not being achieved.

MOSTYN uses tools to achieve their objectives. Such tools include the website and online presence, retail activities and public engagement events. Success at deploying these tools assists the achievement of outcomes. However, it is also possible to use CHAT to look at audience behaviour. Other people - audiences, stakeholders, and the public - have expectations and assumptions about MOSTYN's subject and object. It is these assumptions on which they base their willingness to engage with the organisation. When MOSTYN staff member is told "contemporary art's not for me" from a visitor, they are assessing MOSTYN's subject and object as not something that is relevant or interesting to them.

Visitors also use different tool sets; they might use MOSTYN's tools but are just as likely to use their own. Within an art gallery, particularly a contemporary one, visitors are asked to construct meaning to engage with the art. They do this through their individual tool set: prior knowledge, the people they are with, labels, signage, books, and increasingly their smart phones.

Engeström's (1987) activity system framework based on CHAT took into consideration more contextual factors. 


\section{TOOLS}

Mediate between subject and object

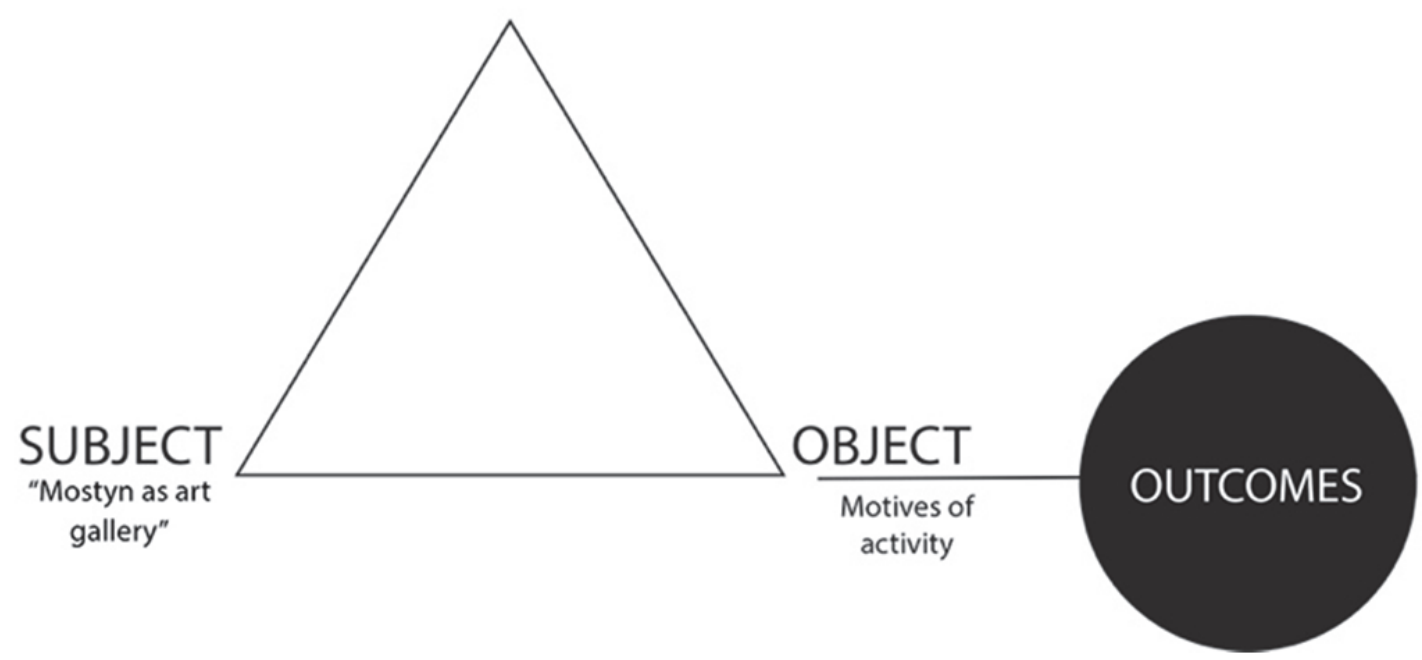

Figure 1: Cultural Historical Action Theory (Vgotsky 1978).

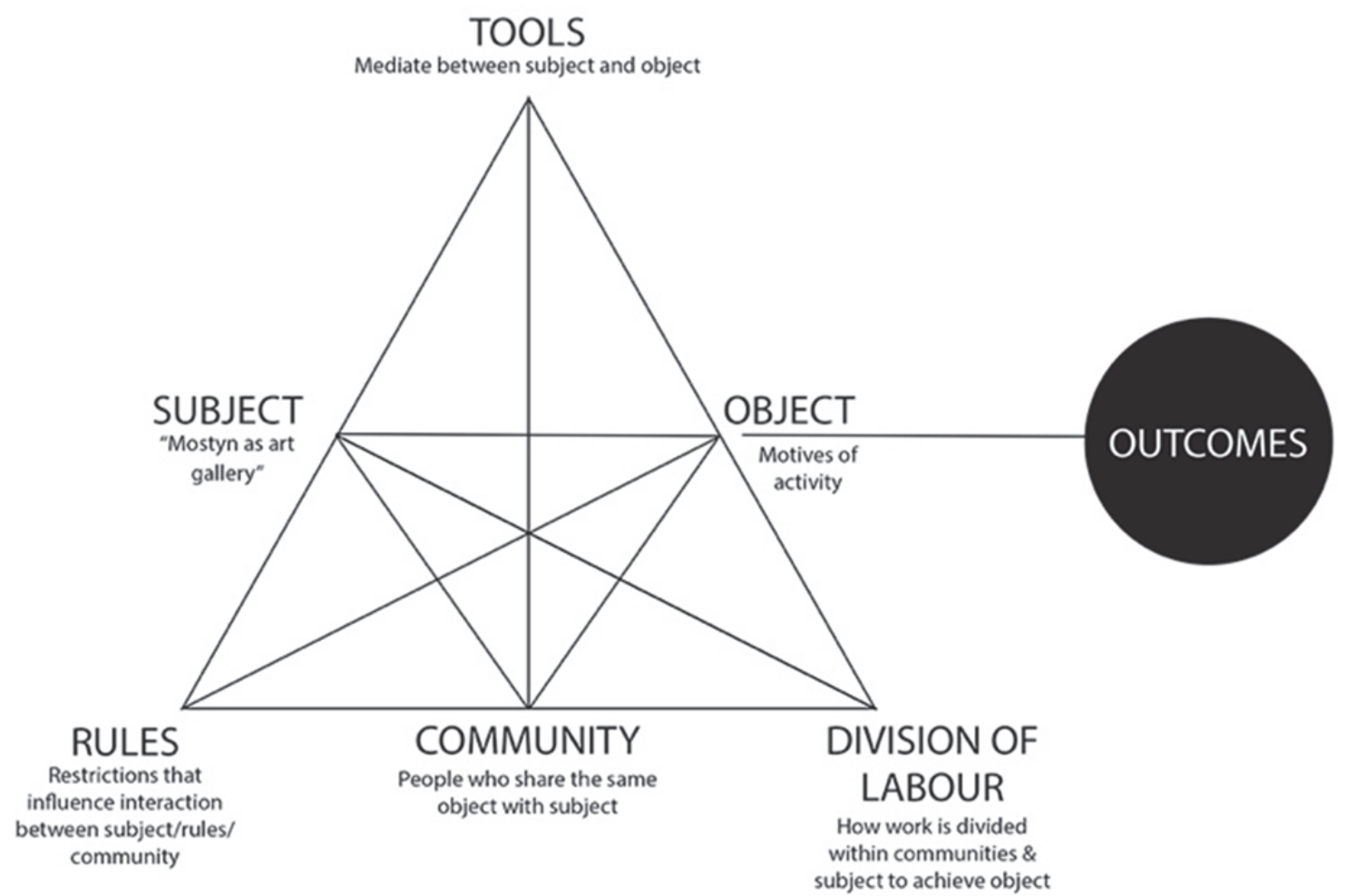

Figure 2: MOSTYN analysed as an activity system (Engeström 1987). 


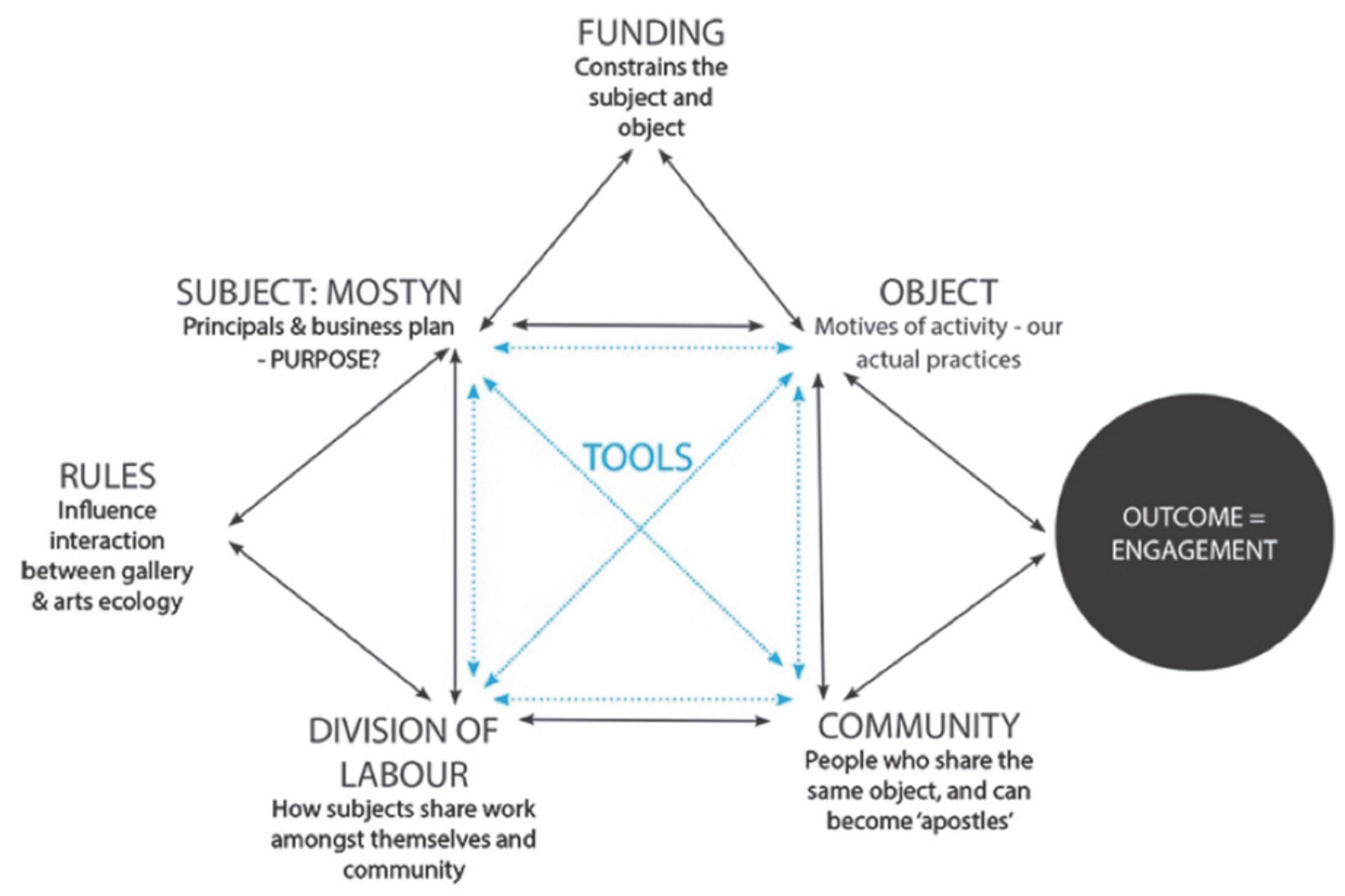

Figure 3: Proposed experimental Activity System 1 (Harding 2018).

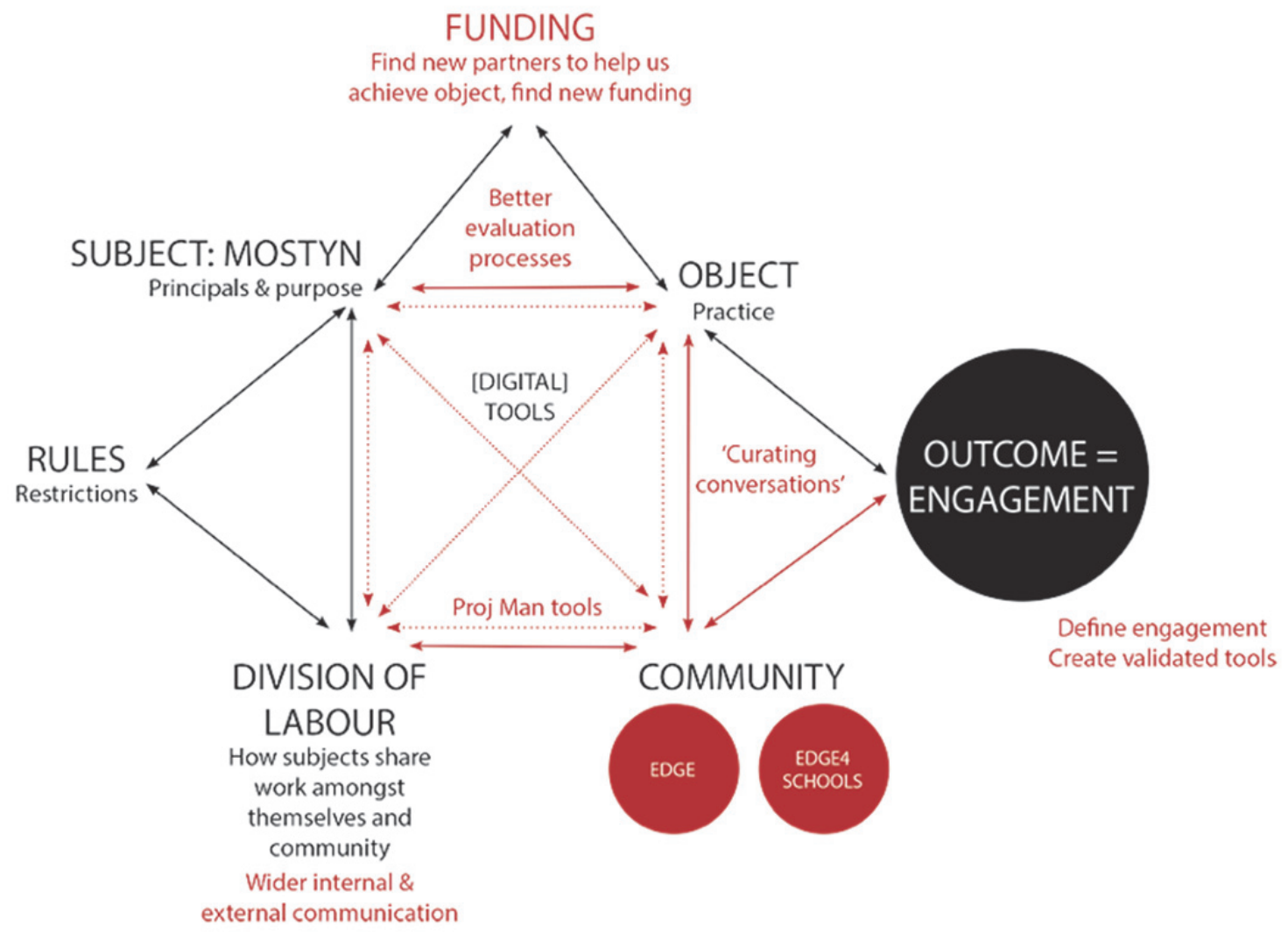

Figure 4: Opportunities for digital interventions within proposed experimental Activity System (Harding 2018). 
As well as a system of subject/object/tools, if we analyse MOSTYN as an activity system then we can consider the wider aspects of rules, community and division of labour. These aspects take account of public diversity and the instability of the context of 'publicness', considering government policy, cultural theory, and communication technology as all aspects which influence public interaction.

Community takes accounts of the fact that the gallery is part of Llandudno, but also of part of communities with makers, artists and other cultural organisations. MOSTYN must therefore recognise that the gallery's meaning is partly generated in relation to all these communities. What is MOSTYN to other people?

Division of labour takes account of how the purpose of MOSTYN is delivered - internally and within wider communities and cultures, supported by political bodies and all under increasing financial pressure.

Rules are those aspects, which influence the interactions between subject, object and community (e.g. the law, health and safety regulations, organisation remits from the Arts Council of Wales), which set the terms of our engagement with communities and shape our subject and object.

However, Harding felt that these maps based on the work of Vgostsky (1978) and Engeström (1987) didn't adequately convey the situation as she perceived it within MOSTYN. She therefore experimented with other ways of mapping activity and created Figure 3: Proposed Experimental Activity System 1.

Funding has become such a significant Rule and MOSTYN's activities are governed by what they are able to secure funding for. Upon discussing this with Director Alfredo Cramerotti, he disagreed and said he did not believe it was so significant that it should be regarded as separately from rules. This therefore remains a point for further debate.

The danger within this situation is that MOSTYN could be defined by "defensive instrumentation" (Belfiore 2012), because the funding context the gallery finds itself means that they can only stage exhibitions which have little or no cost, or they have to justify the cost to a funder and "by articulating value in terms of socio economic impact we fail to recognise that culture is experienced in everyday contexts" (Belfiore 2012). In other words - if you only get funding for big projects, with big demonstrable results, you are failing to support the everyday cultural reasons that people have to visit the gallery.
Figure 3 also reflects the need for tools to work to mediate all elements activity. For example, interaction between subject and community is now mediated by tools such as Facebook. Division of labour/community has improved through the use of social media and 'sharing' to help promote events, and collaborating with other organisations, such as artist collective Culture Action Llandudno, also helps mediate between division of labour and the community. Effectively they are outsourcing promotion to social and physical networks.

Volunteers are the Tools that mediate between the subject and division of labour, as MOSTYN cannot afford to employ more staff and so must rely on some volunteers. Since 2017 there has been a focus on providing more workshops, offering more retail opportunities and more affordable art. These are all tools to assist the gallery to mediate between community and object.

MOSTYN's outcomes, and thus engagement, is therefore heavily reliant upon people external to the organization, which would suggest a high-risk position.

\section{CONTRADICTIONS AND TENSIONS AT MOSTYN}

Once activities have been mapped, we can then look for contradictions, or tensions, within the system "that can hamper or assist in the attainment of the object..." (Yamagata-Lynch 2007)

The key tension with the activity map is that there is no common definition of engagement. Without defining engagement, both in MOSTYN's organisational terms, and from the perspective of audiences, how can MOSTYN define its purpose? Most importantly perhaps, how do MOSTYN's audiences define engagement? What function do they expect MOSTYN to provide for them, particularly in a digital age when a wealth of culture is available $24 / 7$ via just a few taps on a screen?

However, even if the organisation was completely clear on its definition of engagement, and that of its audiences, there are currently no validated tools to measure such engagement. This a gap which Ross (2014) also acknowledges: "There is a lack of consistent practice and standards in the museum computing field in respect of user profiling, motivation, participation and behaviour metrics" citing Dawson et al. (2004) and Haley Goldman and Haley Goldman (2005).

Audience information at MOSTYN is limited to simple footfall door counter and anecdotal information provided by the engagement team. 
Online analytics only provide data on who they are engaging with online.

This means that MOSTYN also misses opportunities to reflect on the findings of such data and establish Key Performance Indicators, which could be used to inform future funding applications or reflect on how events could be advanced.

\subsection{Digital interventions}

Harding's early epistemological reviews indicated that existing research seems often to start with the technology then measure audience reaction to it, for instance, the work of both Hartley (2015) and Ross (2014). The technology leads the process. She therefore identified a crucial gap within both this field of knowledge, and within MOSTYN, to start with audiences and understand their beliefs, behaviours, preferences and insights then, if applicable, use digital means to deal with their identified issues and fulfil the wider organisational ambitions of MOSTYN. In short, this means applying a design thinking approach. She therefore forged a partnership with $\mathrm{Dr}$ Lochrie, with a background in computer science, and Dr Gardiner, and experience designer to pull together the necessary skillset to deliver such a project.

\section{THE EDGE PROJECT METHODOLOGY}

EDGE was co-designed by the research partners to gain insight into what the public want from cultural institutions in a digital age. The first stage of EDGE was a human-centred feasibility study looking at how audiences want to interact with a public art gallery in the face of rapidly developing, culturally competing technology. It would then explore new and authentic ways in which they could communicate with audiences.

Firstly, a staff workshop was held at MOSTYN to role-play and pilot public tasks. A public workshop was then held with 18 audience members, staff, stakeholders facilitated by the research team. Participation was voluntary following a call out via MOSTYN's visitor database and social media. Dr Gradinar designed and led the workshop to deploy and test multiple, agile, lo-fi prototypes (rapidly produced, using cheap materials such as card to test concepts and gain feedback) that participants felt would improve their MOSTYN visitor experience. Participants shared insights about their recent visits, experiences and knowledge of MOSTYN through a series of interactive tasks using a prompts. Findings were then organised and ranked by participants to establish a hierarchy of their needs. They then designed lo-fi prototypes of their ideas using cardboard, paper and string and presented them to the group.
A Cultural Explorer Kit was developed by Drs Lochrie and Gradinar to gain further insight into behaviour within cultural experiences as well as MOSTYN, and types of technological interaction. This was deployed within the gallery from March onwards.

A public consultation was then held within MOSTYN from March 16th onwards presenting the lo-fi prototypes to MOSTYN audiences for further comments, along with a small selection of tasks to triangulate workshop findings. This later included the creation of an interactive 'tree' that used chatbot technology to interact with respondents.

A Design Sprint - which is a rapid, intensive problem solving design exercise - was held in late March with MOSTYN staff to take these public ideas and insight and combine them with organisational need to develop a new digital interface within MOSTYN.

\section{CONCLUSIONS}

The deployment of the Experimental Activity System 1 Framework provided a starting point that allowed Harding to identify broad issues within MOSTYN that could then be explored in further detail through the research process. Further activity analysis, utilising third generation CHAT theory, will be conducted from March 2019 onwards to better understand the inter-relationship of the identified issues.

The research processes needed to be flexible in its approach as it was audience led. It had to allow time to recruit participants, set up workshops, as well as being flexible with locations and the duration of processes to fit in with the busy gallery schedule and availability of the small team. The participants were overwhelmingly positive about the researches processes used. Sometimes difficult, but always constructive, discussion took place with participants and the research team and from this they are continuing to build positive relationships within gallery audiences. The research design proved to be a mutual education process with participants getting insight into how MOSTYN works, especially in the current financial climate.

Early findings are that participants are broadly happy with activities at MOSTYN and support the gallery in its objectives. However, they do want more - more art, more talks, more artists featured, more engagement and interpretation materials and a more significant presence in the town. These now need to be acted upon as an organisation and a series of pilot studies have developed that 
incorporate digital and non-digital cost effective solutions.

The findings to date will be developed into a working interface that will be deployed for a pilot study within MOSTYN from early July until November 2019 and a final report will not only document this process but will provide a toolkit for other cultural organisations looking to develop their own digital tools to enhance audience engagement.

\section{REFERENCES}

Belfiore, E. (2012) "Defensive instrumentalism" and the legacy of New Labour's cultural policies, Cultural Trends, 21(2), pp.103-111. doi: 10.1080/09548963.2012.674750

Dawson, D., Grant, A., Miller, P. and Perkins, J. (2004) User Evaluation: Sharing Expertise to Build Shared Values. In Bearman, D. \& Trant, J. (eds) Museums and the Web 2004: Proceedings. Toronto, p.8. Available at: Archives and Museum Informatics:

http://www.archimuse.com/mw2004/papers/dawson /dawson.html (retrieved 11th March 2019).

Engeström, Y. (1987) Learning by Expanding: An Activity-theoretical approach to developmental research (.pdf). Helsinki, Finland: Orienta-Konsultit.

Haley Goldman, M. and Haley Goldman, K. (2005) Whither the Web: Professionalism and practices for the changing museum. In Bearman, D. \& Trant, J. (eds) Museums and the Web 2005: Proceedings. Toronto. Available at:

http://www.archimuse.com/mw2005/papers/haleyG oldman/haleyGoldman.html (retrieved 11th March 2019).
Hartley, J. A. (2015) Museums and the Digital Public Space: Researching digital engagement practice at the Whitworth Art Gallery. (Doctoral dissertation). Retrieved March 2019 from EThOS database (uk.bl.ethos.677756), p.14

Kaptelinin, V. (2005) The Encyclopaedia of HumanComputer Interaction, 2nd Ed. p.453. Available at: https://www.interactiondesign.org/literature/book/the-encyclopedia-ofhuman-computer-interaction-2nd-ed/activity-theory (retrieved 16th March 2019).

Ross, C. S. (2014) Radical Trust Works: An Investigation of Digital Visitor Generated Content and Visitor Engagement in Museum Spaces (Doctoral dissertation). Retrieved March 2019 from EThOS database (uk.bl.ethos.634698).

Vygotsky, Lev S. (1978) Mind in Society: The Development of Higher Psychological Processes. Cambridge, MA: Harvard University Press.

Walker, D. (2015) Towards the Collaborative Museum? Social Media, Participation, Disciplinary Experts and the Public in the Contemporary Museum (Doctoral dissertation). Retrieved March 2019 from EThOS database (uk.bl.ethos.681251).

Yamagata-Lynch, L. C. (2007) Confronting Analytical Dilemmas for Understanding Complex Human Interactions in Design-Based Research from a Cultural-Historical Activity Theory (CHAT) Framework. The Journal of the Learning Sciences, 16(4), pp.451-484. 